\title{
Underwater detection of dangerous substances: status the SABAT project*
}

\author{
M. SilARSki ${ }^{a}$, P. SibCZYŃSki ${ }^{b}$, Sz. NiedźWIECKI $^{a}$, S. Sharma $^{a}$, \\ J. RAJ ${ }^{a}$, P. MOSKAL ${ }^{a}$ \\ ${ }^{a}$ Institute of Physics, Jagiellonian University, \\ Łojasiewicza 11, 30-348 Kraków, Poland
}

${ }^{b}$ National Centre for Nuclear Research, Soltana 7, 05-400 Otwock, Poland

The Neutron Activation Analysis (NAA) plays an exceptional role in the modern nuclear engineering, especially in detection of hazardous substances. However, in the aquatic environment, there are still many problems to be solved for effective usage of this technique. We present status of SABAT (Stoichiometry Analysis By Activation Techniques), one of the projects aiming at construction of an underwater device for non-invasive threat detection based on the NAA.

PACS numbers: P82.80.Jp, 89.20.Dd

\section{Introduction}

In twenty-first century the risk of terrorists attack is constantly growing around the world. This situation put us in a need to develop more effective methods to detect potential threats and smuggling of illicit materials, e.g. explosives or drugs. New methods are needed particularly for shores and ports protection and monitoring. They are very important also in view of environmental protection of sea areas of intensive warfare, e.g. Baltic Sea [1, 2]. Over 250 kilotons of munition were sunk into Baltic Sea, mostly explosives, but also many chemical agents. It was estimated that if only $16 \%$ of the sunken ammunition was released into the Baltic the life in the sea and at its shores would be entirely ruined for the next 100 years [3]. Precise knowledge of location and amount of these hazardous substances is crucial for a proper planning to deal with such ecological bomb.

* Presented at $2^{\text {nd }}$ Jagiellonian Symposium on Fundamental and Applied Subatomic Physics (Kraków, June 3 - 11, 2017). 
The presently used methods of detection are based on sonars followed by divers inspection, which is not effective and rather expensive and puts the divers into danger due to unknown composition of the suspected items found by the sonar. One of the methods which has a big potential to substitute or support sonars is the Neutron Activation Analysis (NAA). It is based on fast or thermal neutron beams which excite nuclei of investigated substance. Detection of characteristic gamma quanta emitted in de-excitation of the nuclei allows one to identify the stoichiometry of the substance and determine if it is dangerous [4, 5]. There are several designed and produced devices utilizing the NAA for detecting dangerous substances on the ground [6, 7, 8, 9, 10], but in the water one needs to overcome many difficulties connected to neutron attenuation and huge background from oxygen and hydrogen. This background can be reduced for example by using neutron generators with associated $\alpha$ particle measurement and by decreasing the distance between the inspected object and the detector [1]. There are also solutions based on low energy neutrons which are moderated in water before reaching the tested object. The detector is then registering gamma quanta originating from thermal neutron capture [3].

In this articles we present status of the design of a NAA-based device within the SABAT project, where we use guides for the neutron beam and gamma quanta emitted towards the detector. This method not only reduces the background from water but also may provide detection of dangerous substances hidden deep in the bottom of the sea and may allow determination of the density distribution of the dangerous substance in the tested object. Detailed description of the project can be found in [11] and [12].

\section{Preliminary MCNP simulations of the SABAT detection system}

To design and optimize the SABAT detector in terms of high sensitivity and interrogation time we have performed Monte Carlo simulations using the general Monte Carlo N-Particle Transport Code (MCNP) package [13]. We studied geometry of the system including the relative positions of gamma quanta detector and the neutron generator, and the optimal guides dimensions. Moreover, one needs to determine the optimal material composition of the whole device. The simulated setup is shown in Fig. 1. An submarine (blue rectangle) is positioned above the seabed (in yellow) inside which there is an container with hazardous material. The gamma quanta detector and neutron source (generator) are placed inside the submarine and are connected to guides filled with air. Since we optimize the sensor for the detection of war remnants, in particular chemical agents, we assume that the simulated shell contains mustard gas $\left(\mathrm{C}_{4} \mathrm{H}_{8} \mathrm{Cl}_{2} \mathrm{~S}\right)$. The generator emits 


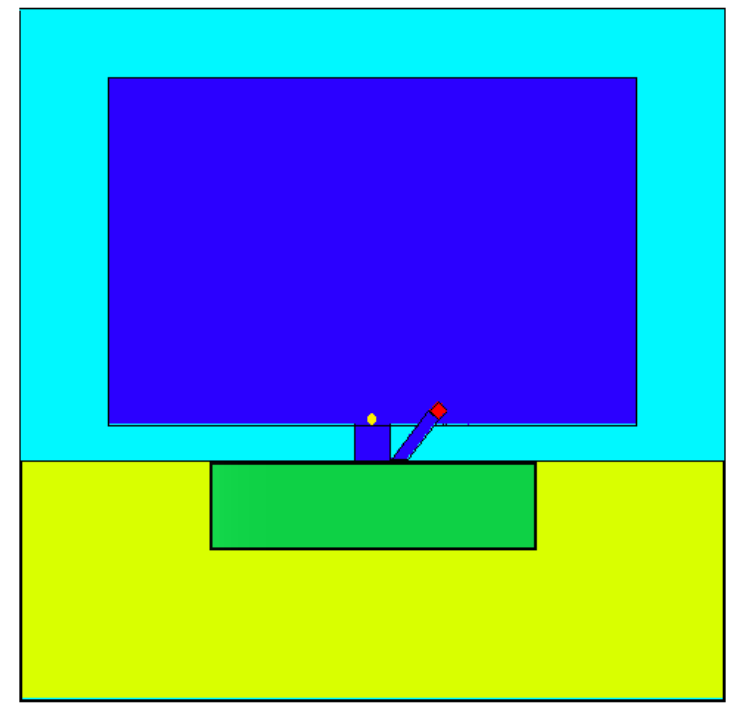

Fig. 1. Exemplary scheme of the simulated SABAT sensor geometry. An submarine (blue rectangle) with dimensions $300 \times 300 \times 200 \mathrm{~cm}^{3}$ contains the neutron source (yellow dot) and gamma quanta detector (red). The neutron and $\gamma$ quanta guides (also in blue) are simulated as $20 \times 20 \times 10 \mathrm{~cm}^{3}$ cuboid and $26 \mathrm{~cm}$ long polyhedron with $20 \times 7.56 \mathrm{~cm}^{2}$ and $16.7 \times 20 \mathrm{~cm}^{2}$ bases, respectively. An container with mustard gas with dimensions $194 \times 50 \times 50 \mathrm{~cm}^{3}$ (in green) is placed inside the bottom of the sea represented by yellow rectangle $\left(400 \times 400 \times 151.5 \mathrm{~cm}^{3}\right)$. Both the submarine and guides are filled with air under normal pressure.

neutrons isotropically and only a small fraction which travels inside the neutron guide reaches the interrogated item without any interaction. These neutrons may activate the mustard gas via inelastic scattering or neutron capture which results in emission of the characteristic gamma quanta which are eventually registered by the detector. It is usually a semiconductor detector, e.g. silicon or HPGe (High Purity Germanium), since it is characterized by very good energy resolution and detection efficiency. On the other hand, they need a cooling system which limits mobility of the whole device. Moreover, HPGe detectors are known to be sensitive to neutron flux, which results in a degradation of the energy resolution over time. As an alternative material one usually uses scintillators. Among many commercially available scintillator materials we have considered so far BGO, $\mathrm{NaI}(\mathrm{Tl})$ and $\mathrm{LaBr}_{3}(\mathrm{Ce})$. BGO is more efficient than the other two materials due to high density, but it has worst energy resolution [14]. The best 
choice, according to Refs. [14, 15], is the $\operatorname{LaBr}_{3}(\mathrm{Ce})$ with a good energy and time resolutions and providing detection efficiency close to the one of HPGe detectors. Exemplary energy spectra of the activated mustard gas obtained with $10^{8}$ generated neutrons and assuming the gamma quanta detector made out of $\mathrm{NaI}(\mathrm{Tl})$ and $\mathrm{LaBr}_{3}(\mathrm{Ce})$ are shown in Fig. 2 a) and b), respectively.In the simulations we assumed that the neutron generator works in continuous beam mode. As expected for both distributions we observe huge background with oxygen peaks around 5-6.5 MeV. The sulfur line at $2.12 \mathrm{MeV}$ is completely covered by very close hydrogen peak. Similarly, the 4.4 MeV line of carbon is overwhelmed by the background, mostly by the 4.5 $\mathrm{MeV}$ characteristic $\gamma$ quanta of silicon [16, 17. We see instead small peaks from chlorine at $0.79,1.16,1.94 \mathrm{MeV}, 7.42,7.80$ and $8.58 \mathrm{MeV}$. As one can see the $\mathrm{NaI}(\mathrm{Tl})$ detector does not provide a clear $\mathrm{C}$ peak and the Chorine lines are much more smeared. The simulations indicate that the performance of this detector could be improved by increasing its size but the identification of potential threats will be anyway much more difficult than in the case of $\mathrm{LaBr}_{3}(\mathrm{Ce})$. To decrease the background from water we introduced a $5 \mathrm{~cm}$ thick led shield around the gamma quanta detector, leaving open only the face connected to the $\gamma$ quanta guide. Unfortunately we did not see any improvement in the sensitivity of the simulated sensor. The performed simulations allowed us also to analyze time structure of the activation processes during the interrogation of the suspected object. In general there are two gamma quanta groups contributing to the energy distributions measured by the detector. First group consists of the prompt gammas hitting the detector within tens of nanoseconds after the neutron emission, depending on the distances between the activated object, neutron source and the detector. These gamma quanta originate mostly from the nucleus excitation by the inelastic scattering. The other group of gammas is registered much later and comes from the thermal neutron capture. The 14.1 MeV neutrons need to be moderated before they can be captured which takes relatively long time. Fig. 3 a) shows the energy spectra of gamma quanta registered by the detector in the $50 \mathrm{~ns}$ time window after the neutron emission for the mustard gas and background. Surprisingly one can observe noise reduction in the energy region of the sulfur peak. Moreover, it turns out that our detection system is even sensitive to another $\mathrm{Cl}$ line in this energy range. The $4.4 \mathrm{MeV}$ line of carbon is unfortunately again merged with Si line. Chlorine is visible also for delayed $\gamma$ quanta shown in Fig. 3 b) which were registered $2 \mu$ s after neutron emission. This time window provides excellent selectivity of many $\mathrm{Cl}$ lines $(1.16,7.42,7.80$ and $8.58 \mathrm{MeV})$. 
a)

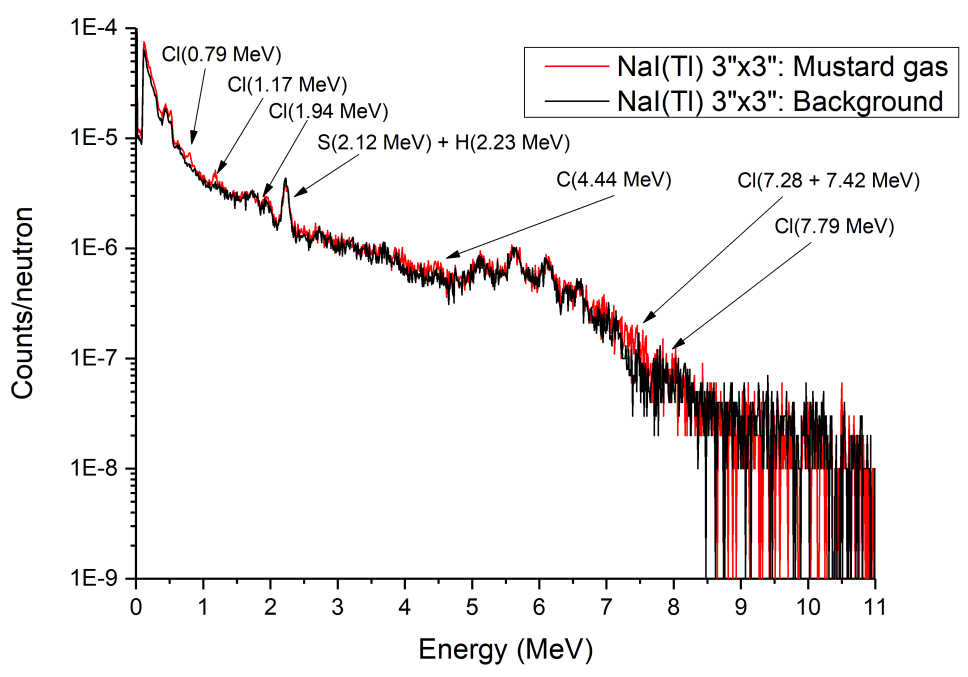

b)

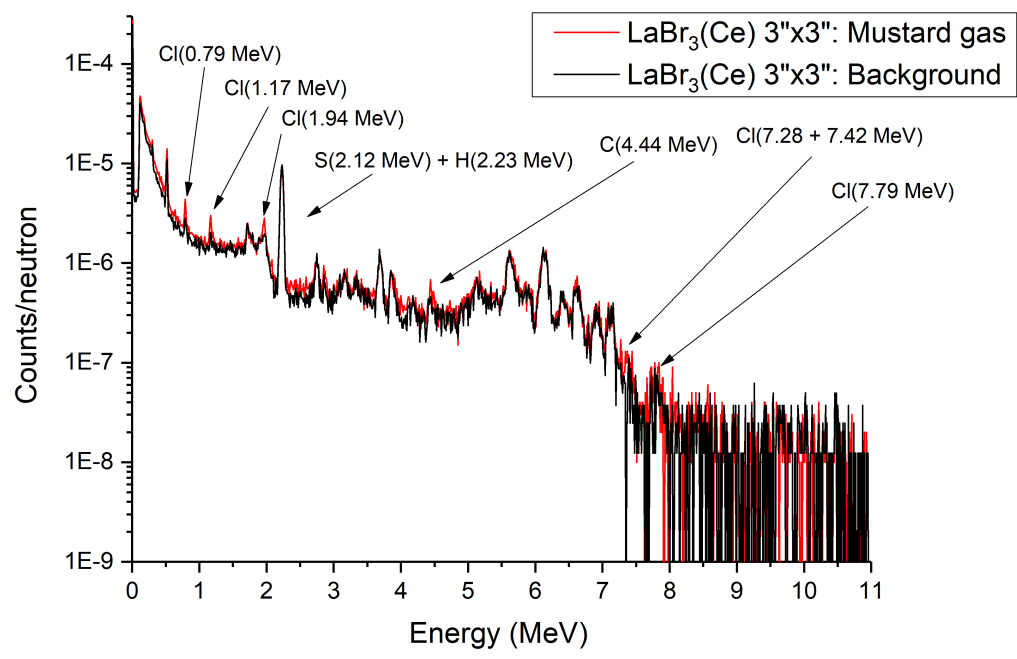

Fig. 2. Energy distributions simulated assuming the $\gamma$ quanta detector made from a) 3" x3" $\mathrm{NaI}(\mathrm{Tl})$ and b) 3" $\mathrm{x} 3$ " $\mathrm{LaBr}_{3}(\mathrm{Ce})$. The black curve represents simulations with mustard gas container while in red we present background simulation when the gas is replaced by sand. 
a)

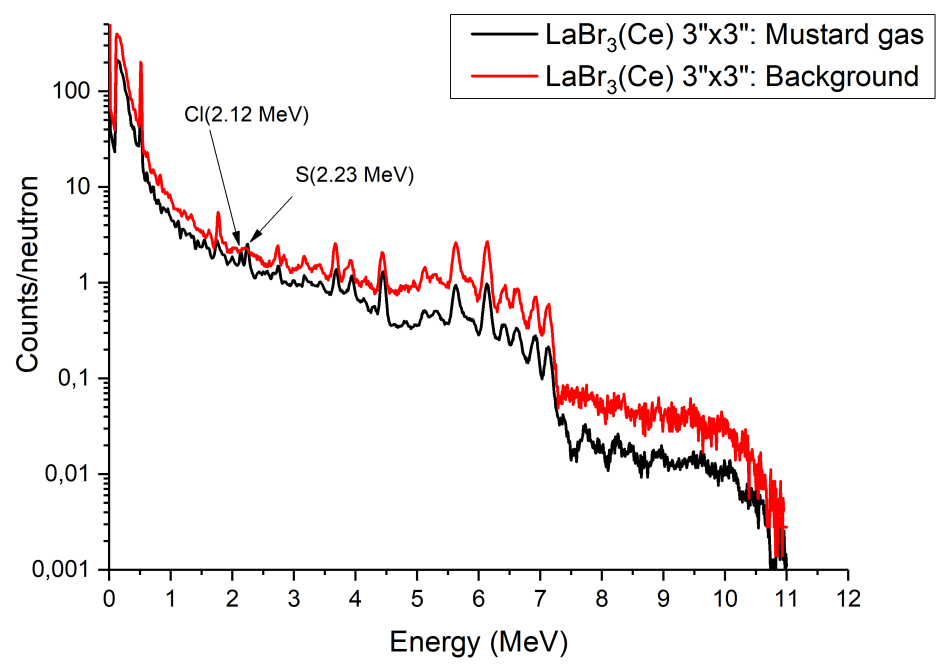

b)

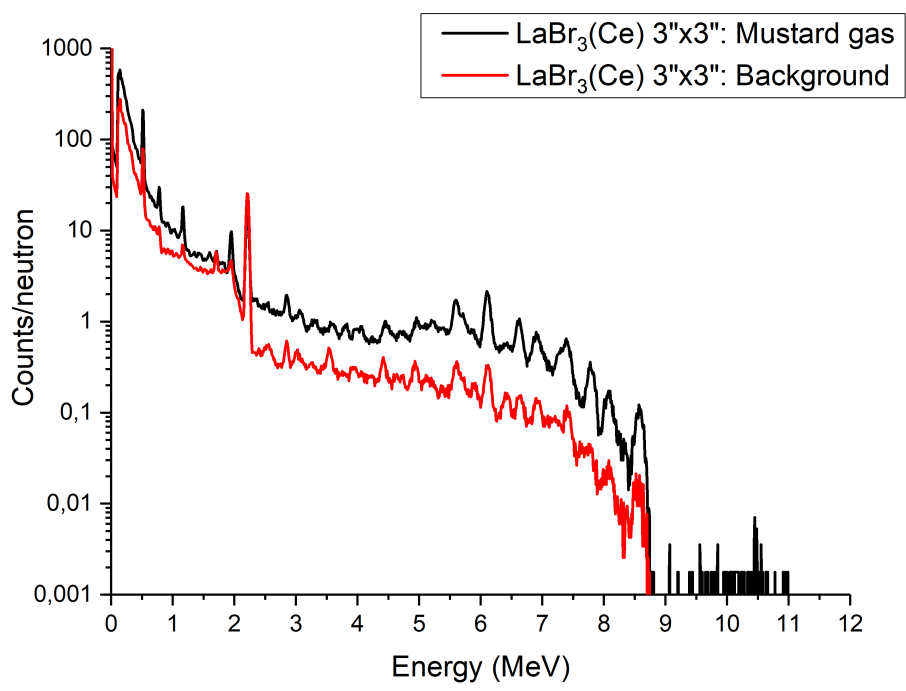

Fig. 3. Energy distributions simulated assuming the $\gamma$ quantum registration time a) within $50 \mathrm{~ns}$ after neutron emission and b) bigger than $2 \mu$ s after neutron emission. The detector is simulated as $3 " \mathrm{x} 3$ " $\operatorname{LaBr}_{3}(\mathrm{Ce})$ scintillator. The black curve represents simulations with mustard gas container while in red we present background simulation when the gas is replaced by sand. 


\section{Conclusions and outlook}

We have been developing a new system for underwater threats detection based on the Neutron Activation Analysis. This device has been optimized in particular to detect chemical agents like mustard gas, soman, sarin etc. To find the best geometry and materials to build the sensor we have performed Monte Carlo simulations using the MCNP software. The first preliminary results show that despite large environmental noise from the water and sand on the bottom of the sea we are able to detect the signature of the hidden mustard gas. According to the simulations, the characteristic lines of chlorine are well visible at low energies, while carbon and sulfur are overwhelmed by background. It turns out that the energy resolution of $\mathrm{NaI}(\mathrm{Tl})$ detector is to poor to give satisfactory results. Thus, the best material to replace semiconductor detector qualified to be $\mathrm{LaBr}_{3}(\mathrm{Ce})$ providing not only good energy and time resolutions, but also reasonable detection efficiency. Analysis of the arrival time distribution of measured gamma quanta shows that even rough time gating gives very promising results. For prompt photons we are able to measure the sulfur and chlorine lines originating from the mustard gas. Unfortuntely, the $4.5 \mathrm{MeV}$ line of Si overlap with carbon line around $4.4 \mathrm{MeV}$. The delayed gamma quanta spectrum shows excellent selectivity of many $\mathrm{Cl}$ lines $(1.16,7.42,7.80$ and $8.58 \mathrm{MeV})$. Altogether, we are sensitive to at least two elements of mustard gas which may provide stoichiometry identification. As it was mentioned before, the noise originating from the environment can be significantly reduced by the requirement of the coincident detection of the alpha particles which are produced together with neutrons [11. The $\alpha$ particle detection and its registration time measurement will be included as the next step in the simulations. This will allow for a final design of the SABAT sensor.

\section{Acknowledgments}

This work was supported by the Polish Ministry of Science and Higher Education through grant No. 7150/E-338/M/2017 and by the National Centre for Research and Development through grant No. LIDER/17/0046/L$7 / 15 / \mathrm{NCBR} / 2016$.

\section{REFERENCES}

[1] V. Valkovic, D. Sudac, K. Nad, J. Obhodas, D. Matika, R. Kollar (2010). "'Surveyor": An Underwater System for Threat Material Detection. International Atomic Energy Agency (IAEA): IAEA.

[2] T. Kasperek, Czas Morza 1, 15 (2001) 
[3] M. Silarski, D. Hunik, M. Smolis, S. Tadeja and P. Moskal, Acta Phys. Polon. B 47, 497 (2016)

[4] P. Moskal, Annales UMCS, Physica 66, 71 (2012).

[5] M. Silarski, Acta Phys. Pol. B Suppl. 6, 1061 (2013).

[6] http://phoenixnuclearlabs.com/solution/explosives-detection/

[7] B. C. Maglich, AIP Conf. Proc. 796, 431 (2005); http://www.calseco.com/

[8] http://www.euritrack.org/

[9] http://www.sodern.com/

[10] Ł. Kaźmierczak et. al., Acta Phys. Polon. A 127, 1540 (2015); http://aid.ncbj.gov.pl/index.php/en/products/swan

[11] M. Silarski, D. Hunik, P. Moskal, M. Smolis, S. Tadeja, Acta Phys. Polon. A 127, 1543 (2015).

[12] M. Silarski, P. Moskal, patent number: P 409388, PCT/PL2015/050021

[13] T. Goorley et al., Nuclear Technology 180, 298 (2012).

[14] P. A. Russo, D. T. Vo, PANDA 2007 Addendum, LA-UR-05-3813, 5 (2007).

[15] C. Eleon, B. Perot, C. Carasco, Nucl. Instrum. Meth. A 619, 234 (2010).

[16] M.B. Chadwick et al., Nucl. Data Sheets 112(12), 2887 (2011).

[17] M. R. Bhat, Nuclear Data for Science and Technology (revised as of April 2014) edited by S. M. Qaim (Springer-Verlag, Berlin, Germany, 1992). 